\title{
Parametri ipocentrali dai dati macrosismici del terremoto del Friuli, Maggio 1976
}

\author{
(Hypocentral parameters of macroseismic data of Friuli \\ earthquake, May 1976)
}

\author{
C. Gaspirini *
}

Ricevuto il 12 Ottobre 1976

RIAssunTo. - Nel presente lavoro sono riportati succintancente i dati materosisuiei del terremoto del Friuli del a Maggio 197 (i. I risultati oftenuti somo stati confrontati con quelli analitici.

Sumars. - In this paper are briefly reported macroseismic data of the Friuli (Italy) earthquake (6 May, 1976). The theoretical results have been compated with the experimental ones.

\section{PREMESSA}

I dati macrosismici sono stati acquisiti tramite diverse fonti; da sopraluoghi effettuati dallo scrivente dal 14 al 25 Maggio 1976, dalle cartoline macrosismiche dell'Ufficio Centrale di Meteorologria, ed in ultimo controllati ed ampliati con i dati gentilmente forniti dal "Servizio Sismico " del Ministero dei Lavori Pubblici e dall'tfficio Tecnico del Commissario Straordinario di Governo ad Eiline.

* Istituto Nazionale di Grofisica. Osservatorio (ieofisieo Contrale. Monte Porzio Catone Roma. 
Tra le numerose scale macrosismiche utilizzabili, la più comunemente usata in Europa è la MCS (Mercalli, Cancani, Sieberg); tale scala in questo specifico caso risulta più appropriata delle altre, nella descrizione dinamica degli eftetti di questo sisma, data la situazione urbanistica locale del Friuli e delle aree limitrofe.

Ise isosiste riportate in fig. 1, sono appunto disegnate in tale scala.

Il grarlo cl'intensità di un sisma è ottenuto dal confronto tra gli efletti osservati e la scala di paragone, e non ha un significato matematico preciso ma è solo un indice gerarchico dei danni causati dal terremoto.

La valutazione dell'intensità per i gradi VIII, IX, $\mathrm{X}$, va eflettuata prevalentemente in base agli effetti sugli edifici. Nella scala da noi usata il X grarlo è caratterizato da distruzioni a circa il $75 \%$ degli erlifici, il $50 \%$ er il $25 \%$, per i gracli successivamente inferiori.

L'area epicentrale della scossa del 6 Maggio ha un'estensione di circa $900 \mathrm{kmq}$ e comprende gli abitati di Gemona, Venzone, Osoppo, Trasaghis, Maiano, Borlano, Forgaria, i quali hamno ma percentuale di damno compresa tra $80 \%$ ed il $65 \%$.

Numerosissime sono state le frane sia nell'area epicentrale che all'esterno di essa; particolarmente interessati sono stati i fronti perlemontani che vanno da Artegna a Venzone, e da Forgaria a Bordano.

Altri tipi di dami meno caratteristici, per la valutazione del grado, si sono pur essi verificati come quelli all'acquerlotto del Natisone, e quelli agli assi delle turbine nella Centrale in roccia di Somplago. Dissestamenti alle strade si sono verificati sia con carattere isolato che omogeneamente per alcuni tipi litologici.

Tenendo conto degli efletti dei dami nel loro insieme, l'intensità macrosismica massima è valutabile come "rovinosa", ció̀ pari al X grarlo della MCS, equivalente arl un'accelerazione di circa 243 $\mathrm{cm} / \mathrm{sec}^{2}$.

Ia valutazione macrosismica nelle scale MSK (Nedverler, Sponhener, Karníli), ed MII (Mercalli morlificata), tiene conto del tipo di erlifici damneggiati, per cui ì inclispensabile un censimento dettagliato dei ramni subiti dagli edilici " tipo" considerati nelle scale. In assenza di questo, in linea di massima, l'intensitì è valutabile dalle relazioni intercorrenti tra intensità er accelerazione, fig. 2. Risulta che per il $\mathrm{X}$ grarlo della MCS corrisponde un IX nella MSK e nella MII. 


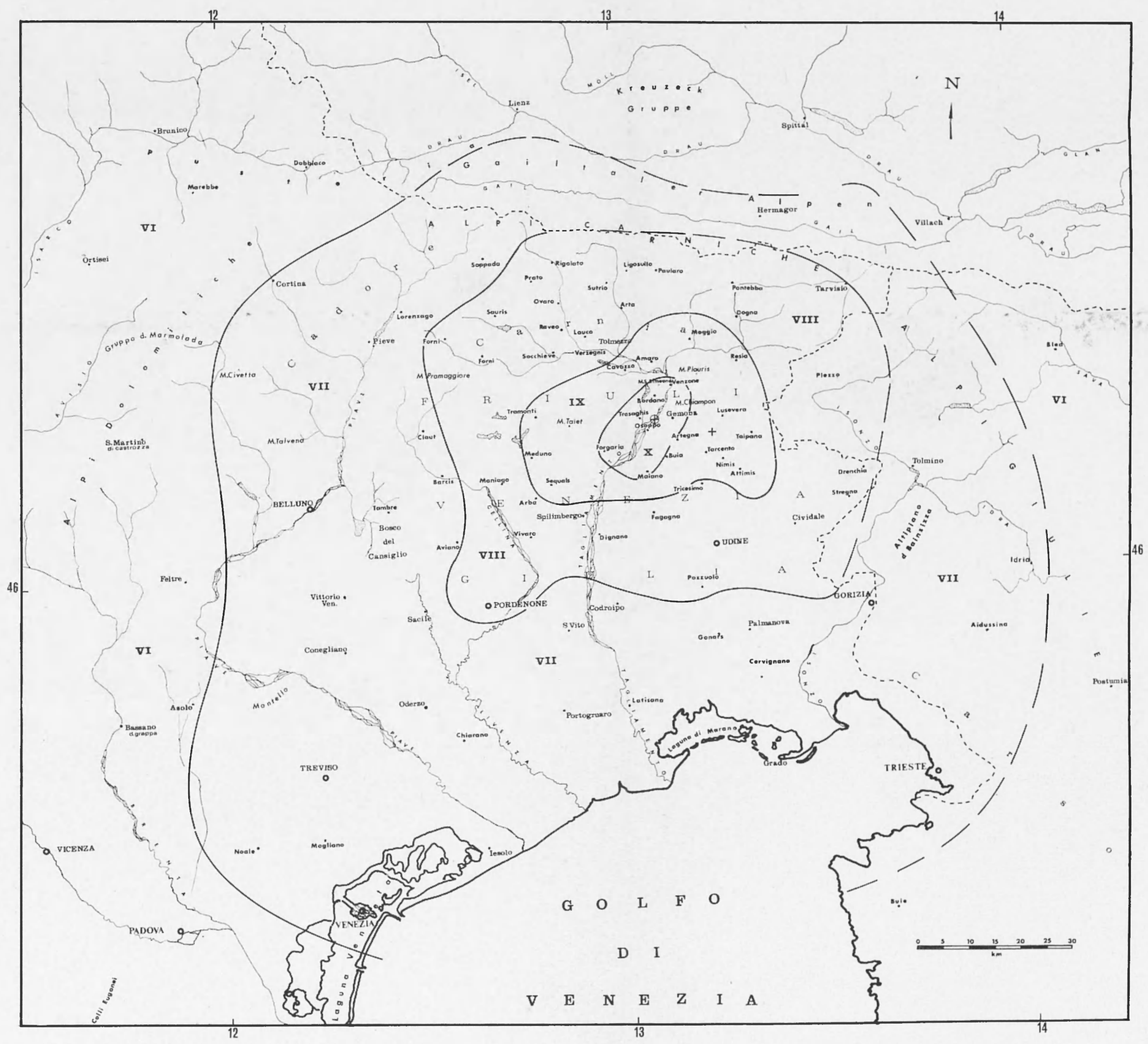

Fig. I - Isosiste del terremoto del Friuli del 6 Maggio 1976, i gradi macrosismici sono riportati nella scala MCS. Il cerchio crociato è la locazione dell'epicentro macrosismico, mentre la crocetta corrisponde alla posizione dell'epicentro analitico. 
Il sisma si è manifestato con la seguente intensità:

$\mathrm{X}$ a Bortano, Forgaria, Gemona del Friuli, Maiano, Osoppo, Trasaghis, Venzone,

IX a Artegna, Attimis, Buia, Cavazzo, Lusevera, Meduno, Moggio Udinese, Nimis, Resia, Sequals, Taipana, Tarcento, Tramonti di Sopra, Tramonti di Sotto,

VIII a Tricesimo, Pozzuolo, Fagagna, Dignano, Spilimbergo, Vivaro, Arba, Porkenone, Maniago, Forni di Sopra, Forni di Sotto, Socchieve, Sauris, Verzegnis, Lauco, Raveo, Tolmezzo, Prato Carnico, Ovaro, Arta, Sutrio, Rigolato, Ligosullo, Pontebba, Dogna, Tarvisio, Drenchia, Stregna, Cividale del Friuli, Udine, Paularo,

VII a Portogruaro, S. Vito al Tagliamento, Chiarano, Sacile, Iesolo, Oderzo, Treviso, Conegliano, Vittorio Veneto, Mogliano, Venezia, Noale, Tombre, Barcis, Aviano, Claut, Belluno, Pieve di Carlore, Lorenzago, Cortina, Sapparla, Trieste, Gorizia,

VI a Palmanova, Cervignano del Friuli, Gonars, Grado, Codroipo, Latisana, Parlova, Vicenza, Bassano rel Grappa, Marostica, Asolo, Feltre, S. Martino di Castrozza, Ortisei, Dobbiaco, Marebbe, Brunico.

\section{EPICENTRO}

L'epicentro macrosismico ì stato posto grosso modo al centro dell'area parossistica tra Trasaghis ed Osoppo, nel punto di coordinate:

latitudine $46^{\circ} \quad 16^{\prime}$ Nord, longitudine $13^{\circ} \quad 06^{\prime}$ Est.

Analiticamente ì stato ottenuto ( $\left.{ }^{(}\right)$,

$$
\varphi=46^{\circ} 15^{\prime} 11^{\prime \prime} \mathrm{N}, \quad \lambda=13^{\circ} 14^{\prime} 24^{\prime \prime} \mathrm{E} .
$$

\section{PROFONDIT $\lambda$}

Il calcolo della profondità, $h$, effettuato con la relazione:

$$
h=\frac{r_{n}}{\sqrt{\frac{I_{0}-I_{n}}{3}}}
$$

in cui $r_{n}$ è il raggio merlio dall'epicentro all'isosisma di grado $I_{n}, I_{0}$ l'intensità macrosismica all'epicentro, ha clato i seguenti valori: 
C. GASPARINI

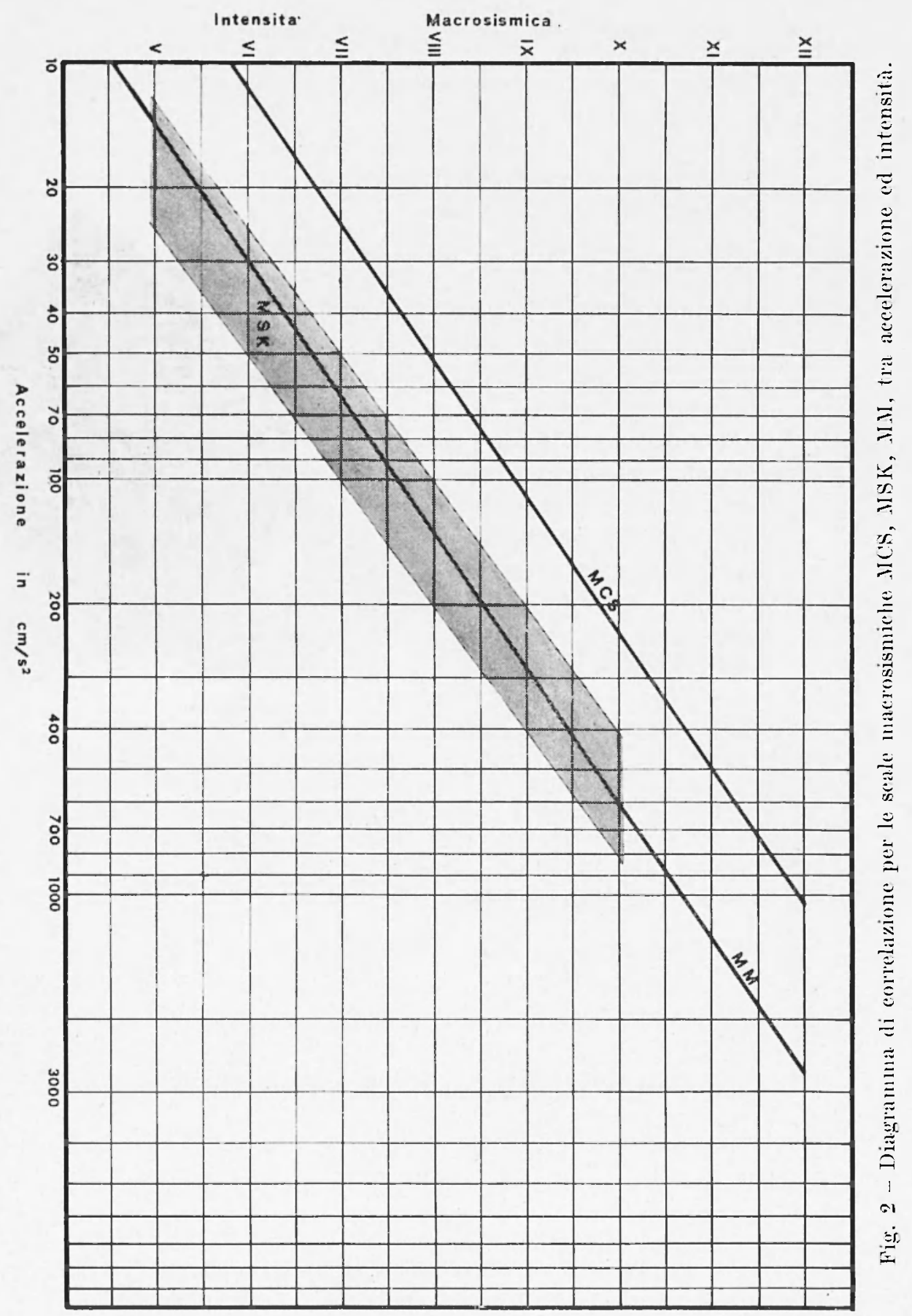




$$
\begin{array}{cll}
\text { per } & I_{1}=\mathrm{IX} & h=18,3 \mathrm{~km} \\
" & I_{2}=\mathrm{VIII} & h=22,3 \mathrm{~km} \\
" & I_{3}=\mathrm{VII} & h=25,3 \mathrm{~km} \\
" & I_{4}=\mathrm{VI} & h=18,0 \mathrm{~km} \\
" & I_{5}=\mathrm{V} & h=22,3 \mathrm{~km}
\end{array}
$$

dai valori precerlenti deriva come media delle profondità

$$
\bar{h}=21 \pm 1 \mathrm{~km} \text {. }
$$

Analiticamente, i valori ottenuti variano a seconcla del modello crostale nsato (Console, Gasparini 1976) (1) da 1 a $40 \mathrm{~km}$.

\section{Magitudo}

La relazione per il calcolo della magnitudo macrosismica regiomale (Gasparini, Giovani, 1973) $\left({ }^{2}\right)$ ì:

$$
\mathrm{M}^{* *}=0,+37 I_{0}+0,874 \log h+0, \pi 14
$$

dalla quale si ottiene per $\bar{h}=21 \mathrm{~km}, Y^{*}=6.2 f \simeq 6.2$, mentre le magnitudo strumentali locali fornite dai vari osservatori sono comprese tra i 6.2 e 6.5 .

\section{A'TEETAZIONE MACROSISMICA}

Il coeinciente di attenuazione macrosismica, non is altro che la trasposizione numerica clella variazione di grado (inclice) in funzione della distanza. Essa c, come l'intensità macrosismica, fortemente influenzata dalla natura geologica delle formazioni attraversate dallonda sismica, quindi ì soggetta a notevoli variazioni in caso di eterogeneità geologica.

Il coefficente di attenuazione a, dell'energia macrosismica ì stato calcolato tramite la relazione:

$$
u=\frac{2,30}{D_{n}--h_{n}} \log \frac{I_{0}}{I_{n}}
$$

in cui $I_{n}$ ¿ la distanza ipocentrale, $h$ la profondità, $I_{0}$ il grarlo allepicentro, $I_{n}$ il grado dell'isosista consirlerata.

Nel caso in esame sono stati presi in consiclerazione alcuni percorsi significativi.

Dallepicentro verso nord, perpendicolarmente alla direttrice alpina: 


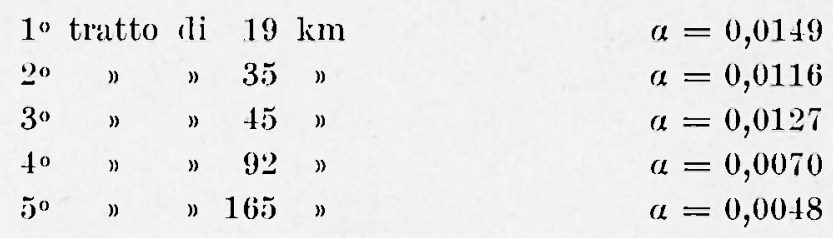

attenuazione media 0,0102 노 0,0037 .

Verso sul, in clirezione opposta alla precelente:

$$
\begin{aligned}
& 10 \text { tratto di } 15 \mathrm{~km} \\
& \alpha=0,0228 \\
& \text { go " } " 35 \text { " } \quad \alpha=0,0116 \\
& 3^{\circ} \text { " " } 100 \text { " } \alpha=0,0044
\end{aligned}
$$

Nella direzione parallela al fronte perlemontano delle $\mathrm{Al}$ pi calcaree mericlionali:

$$
\begin{aligned}
& \text { 10 tratto ali } 15 \mathrm{~km} \\
& \alpha=0,0228 \\
& \text { 20 " " 54 " } \\
& \alpha=0,0061 \\
& 3^{\circ} \text { " } 190 \text { " } \\
& \alpha=0,0050 \\
& \text { 4० " " } 117 \text { " } \\
& \alpha=0,0052 \\
& 5^{\circ} \text { " } 142 " \\
& \alpha=0,0057
\end{aligned}
$$

attenuazione media $0,0089 \pm 0,0031$.

Parallelamente al fronte delle $\Lambda l p i$ Giulie (sistema dinarico):

$$
\begin{aligned}
& 1^{\circ} \text { tratto di } 23 \mathrm{~km} \\
& \alpha=0,0107 \\
& 20 \text { " " } 44 \text { " } \\
& \alpha=0,0082 \\
& 3^{\circ} \text { " } 191 \text { " } \\
& \alpha=0,0050 \\
& 40 \text { " " } 111 \text { " } \\
& \alpha=0,0056
\end{aligned}
$$

attenuazione media $0,0074 \pm 0,0026$.

I ralori riportati, illustrano molto bene le due grosse differenze tra $\mathrm{i}$ percorsi trasversali al massiccio alpino, in cui $\alpha$ è circa 0,01 , e nell'opposta direzione in cui l'energia macrosismica è favorita con $a \simeq 0,008$.

\section{BIBIIOGRAFIA}

(1) Cossold: R., Gasparisi C., 1976. - Analisi dei parametri ipoeentrali del terremolo del Friuli del a Maggio 19ro. "Annali di Geofisica", XXIX, 3.

() Gaspanixi (C, Giovaxi L., 1973. - Allivili sismica in Ilalia nellamo 1970. "Annali di Geofisica", XXVI, $2-3$. 
E

$07^{\mathrm{h}} 50^{\mathrm{m}}$

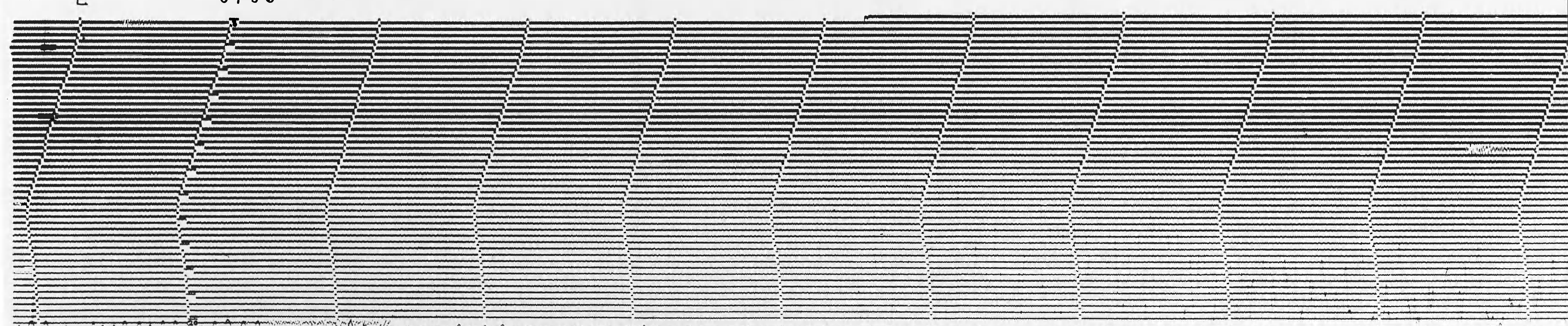

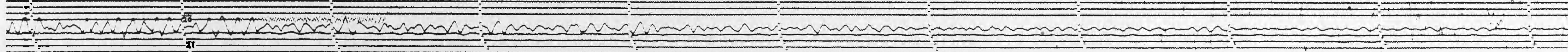

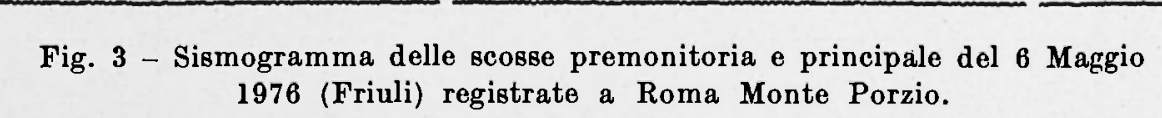

